\title{
A Tailor made System for providing Personalized Services
}

\author{
Mario Casillo \\ DII \\ University of Naples "Federico II" \\ Naples, Italy \\ \{mario.casillo\}@unina.it
}

\author{
Francesco Colace, Francesco Pascale \\ DIIn \\ University of Salerno \\ Fisciano (SA), Italy \\ \{fcolace, fpascale\}@unisa.it
}

\author{
Saverio Lemma, Marco Lombardi \\ SIMASLab \\ University of Salerno \\ Fisciano (SA), Italy \\ \{slemma, malombardi\}@unisa.it
}

\begin{abstract}
Today, the existing technologies, such as smartphone and other pervasive devices, can be used for context awareness and help people to undertake conscious choices. In fact, unlike what happened in the past, all of these data are managed and contextualized for the particular application. We need to understand, select, treat and finally opportunely expose these data. In this scenario, there are many Context Aware applications.

This paper introduces a tailor made system for providing personalized services and it is based on a graphical formalism for the context representation: the Context Dimension Tree.

This system provides the needed information about places that are of great interest for the visitors, selecting them using user preferences. A case study is applied to an event in Salerno, an Italian town, called Artist's Lights. Finally, an experimental campaign has been conducted, obtaining interesting results.
\end{abstract}

Keywords-Context-Aware Computing, E-Citizenship, Adaptive Systems.

\section{INTRODUCTION}

The Italian towns have a cultural heritage that often do not succeed in being completely enhanced. The natural, artistic and cultural resources present in the Italian towns, above all the smallest ones, many times remain hidden and are not enjoyed by the tourists[12]. This problem typology becomes even more important when the tourist has few hours to visit a town: think, for instance, about some passengers of a cruise who in few hours have to visit an unknown place. The problem arises also for those people who, for work, live an experience in a town that they can visit in little time. Where to eat? What to see? How to move? These are the typical questions that such a user makes when he/she is in a station, an airport or a harbor. If in the big towns there are pre-constituted itineraries that can be easily made by the tourists, this is not always true in towns of little or medium dimension that, even if they have a cultural heritage absolutely interesting, often risk of not enhancing it completely.

On second thoughts, information necessary for the enhancement of the resources of a town are, in many cases, already present on the web: the social networks have much information about the resources present in a town. On the other hand, also the public institutions, usually, develop some contents in support of the cultural resources present in the territory, but not present in places not easily reachable by the tourists, above all the foreign ones. Moreover, often, there are also services that can be useful for a tourist who unlikely knows to find them. Therefore, it is necessary to create a framework that can integrate contents and services to support a user inside a certain territorial context [11].

When the main aim is to create applications, devices, and systems that are easy to use, it is essential to understand the context of use. Today, with context-aware computing, it can be possible to consider the situation of use not only in the design process, but in real time while the device is in use. Generally in Human-Computer Interaction (HCI) [8] the main aim is to understand the user and the context of use and create designs that support the major anticipated use cases and user use situations [9]. In Context-Aware Computing on the other hand, making use of context causes a fundamental change: it can supports more than one context of use that are equally optimal. At runtime - when the user interacts with the application - the system can decide what is the current context of use and provide a user interface specifically optimized for this context. With context awareness, the job of designing the user interface typically becomes more complex as the number of situations and contexts which the system will be used in usually increases. In contrast to traditional systems, they are not designed for a single -or a limited set - of contexts of use, but for several contexts. The advantage of this approach is that it can provides optimized user interfaces for a range of contexts [10].

One of the most important part is select and describe all user situation: user's actions taken with his/her mobile device commonly involve directly or indirectly more than the device holder, thus resulting the usage context to have impact on more than just one person. This information are very important in order to understand what actions to take by context awareness applications and provide pervasive and sometimes indispensable indications for the user.

In this paper is described a context-aware approach for promoting tourism events in Salerno, a town in South of Italy. This work will be organized in this way: in the following paragraph, related works are analyzed, especially for the Mobile Context Awareness. In this paper, the purpose is to give an answer to the problem of the context representation using the Context Dimension Tree (CDT) formalism [21]. Then, it will be present a Context Aware 
App able to promoting tourism events. Some experimental results will be described in the last part of the paper.

\section{RELATED WORKS}

Today there are many works in research that focus the attention on Context Awareness, particularly in the mobile applications. As seen previously, an app lends itself well to this type of persasive solutions, because today every person has a smartphone connected to the network and it is possible, using the right tools, get all those useful contextual information that can be used to provide an appropriate recommendation to the user. In this context, the main effort has been to retrieve the contextual data of the event or situation of interest and then treat them adequately in order to provide pervasive services to the users.

In [16], a solution has been proposed: an android widget that recommends apps, that through calls and SMS of user, depending on the day/hour of the week and also based on the past behavioral pattern of user activity. The recommendations can be extremely helpful for the user that finds information quicker and effortless.

In [17] a context-aware mobile language learning application is presented, that it focused on providing language support to users living in foreign countries. It suggests context-relevant vocabulary, considering usage context attributes such as user's gender, geolocation and native language. The application architecture is composed by three components which are able to dynamically acquire and analyze the usage context to provide the corresponding information based on knowledge domain. In this work is introduced the Contextual Language Learning (CoLaLe) application, which was designed to support foreign language learning for overseas students. In [2] a novel method to estimate daylight illumination has been proposed using this information in outdoor augmented reality (AR) applications to render virtual objects with coherent shadows. The illumination parameters are acquired in real time from context-aware live sensor data. The particularity is that the Sun's position is calculated based on the user location and time of day, with the relative rotational differences estimated from a gyroscope, compass and accelerometer.

In [20] a novel solution to overcome problems caused by user movement in the mobile cloud computing context is proposed. To this end, a mobility model and a fault tolerance mechanism for optimizing the offloading decision are introduced. Simulation results showed that this proposal can reduce the execution time of mobile applications. This solution considered different parameters that influence offloading like bandwidth, amount of transmitted data and user mobility, with extension to existence prediction models with the aid of Markov chain to find the optimal solution.

In [19] ClickSmart is proposed: a viewpoint recommendation system that can assist a user in capturing high-quality photographs at well-known tourist locations. ClickSmart can provide real-time viewpoint recommendation based on the preview on the user's camera, current time, and user's geolocation. It makes use of publicly available geotagged images along with the associated metadata for learning a recommendation model.

In [15] a context-aware application is proposed and it provides different user authentication methods that are set up according to the auto-detection of areas designated as safe zones by the user. This application aims to improve the overall security of the content of a given device by securing individual applications.

In [24] the context-aware recommendation approach has been introduced and it integrates network location context, user context and demand context. An extensible metamodel is proposed in this system, thus the description of class property can be scalable. The consistent representation of service and user demand will provide convenience for service matching and service filtering. In order to ensure real-time update of service information, this work puts forward three methods: spontaneous service updating, delayed service updating based on accessing, as well as delayed service updating periodically.

In [3] a novel technique for finger tap detection using the built-in speakers as primary sensors is introduced, named TouchSpeaker. Speakers used as sensors (i.e., as microphones) can overcome the aforementioned limitations; these are passive devices, consuming no power for sensing (except for ADconversion).

In [23] a novel approach to recommend services on mobile devices to user has been proposed. This system is composed by three modules: a user behavior model by taking advantage of user's mobile context information like time and location to describe the user states; a model to explain how the sequential service invocations are generated by analyzing the collected sequential history record of mobile users; a logistic model tree approach to determine user state according to given mobile context information, and recommend services to user according to his user state.

In [22] has been presented context modeling and management approaches intended for the optimization of smart interactions and services, discusses the main challenges and requirements of context-awareness in the smart Internet, and provides a feature-based framework useful for the evaluation and implementation of context modeling and management mechanisms.

In [14] has been presented a Semantic Engine of the AMBIT (Algorithms and Models for Building contextdependent Information delivery Tools) architecture in order to focus on the knowledge management foundations that are using to laying this system.

In the next paragraph, an approach to contextualization contents and services using the Context Dimension Tree will be presented.

\section{CONTENTS AND SERVICES CONTEXTUALIZATION}

For contextualization is meant the act or process of putting information into context; making sense of information from the situation or location in which the information was found. This means that to have the appropriete requests for a context-aware system, it needs having a right model. In order to make contextualized queries, it is necessary to define a model for the representation and management of the context itself, which allows filtering the resources obtained, on the basis of contextual parameters (user position, user profile, user friends, etc.): this operations are made through CDT.

Therefore, the result shows itself like a well-organized information that presents a general introduction about the place reached by the user, according to his/her interests and enriched with the experiences shared by similar users, and a 
list of the main suggested attractions about the near places visited by the friends.

In particular, CDT is used to be able to represent, in a graphic form, all possible contexts that you may have within an application. CDT plays a fundamental role in tailoring the information space according to the user's information needs, as well as an analysis of relevant features of context models. It is thus important to notice that this notion of context is strictly connected to the considered application and is not meant to model the general knowledge concerning one or more areas of interest, a situation where a data schema, or a domain-ontology may be better suited [4, 6, 7].

CDT is a tree composed of a triad $\langle\mathrm{r}$; $\mathrm{N}$; A $>$ where $\mathrm{r}$ indicates its root, $\mathrm{N}$ is the set of nodes of which it is made of and $\mathrm{A}$ is the set of arcs joining these nodes.

A dimension node, which is graphically represented by the color black, is a node that describes a possible dimension of the application domain; a concept node, on the other hand, is depicted by the color white and represents one of the possible values that a dimension may assume. Each node is identified through its type and a label.

The children of the root node $\mathrm{r}$ are all dimension nodes, they are called top dimension and for each of them there may be a sub-tree. Leaf nodes, instead, must be concept nodes. A dimension node can have, as children, only concept nodes and, similarly, a concept node can have, as children, only dimension nodes. In addition to nodes, you can use other elements: the parameters, which may arise both from a dimension node (graphically represented by a white square) and from a concept node (white triangle), submitting them to particular constraints. In fact, a concept node can have more than one parameter, while a dimension node can have only a parameter and only in case it has not already children nodes. The introduction of parameters is due to their usefulness in shaping the characteristics that can have an infinite or very high number of attributes. For example, a node representing Cost dimension risks having a high number of values that should be specified by as many concept children nodes. In a similar case, it is therefore preferred to use only one parameter, whose value will be specified in each case. Leaf nodes, in addition to concept nodes, can also be parameters. In general, each node has a parameter corresponding to a domain, $\operatorname{dom}(\mathrm{nP})$. For parameter nodes connected to concept nodes, the domain can be a set of key values from a relational database, while in case of parameter nodes connected to dimension nodes, the domain is a set of possible concept nodes of dimension.

Therefore, CDT is used to systematically describe the user needs, and to capture the context the user is acting in. It plays a fundamental role in tailoring the target application data according to the user information needs. Once the possible contexts have been designed, each must be connected with the corresponding view definition. In this ways, when the context becomes current, the view is computed and delivered to the user [18].

In fact, through the CDT, it is possible, after analyzing the domain of application, to express the size characteristics and values they can take in a graphical way by, respectively, dimension nodes and concept nodes or parameters.

The assignment to a dimension of one of its possible values is a context element. The context element can be considered the main feature of the application, by which a context can be decomposed. The moment you make the formulation of the context, you must specify all the context elements that are part of it and that enable its creation. Any context is expressible by an "and" combination of the context elements to which they are peculiar.

By definition, you can begin to understand how you will create views based on data relating to each context; in fact, they will be built starting from the portions of the database and then from the partial views, associated to the context element that takes part into context information.

The CDT elaboration is composed of methodologies and phases to obtain contextual resources. The methodology has been realized in order to manage the database and to carry out reductions of their content based on the context. The purpose is to help the designer in the definition of all contexts relevant to the considered application and, later, in the association to each context of the portion of the database containing the relevant data about the context.

The methodology consists of three main phases, which we will see in detail later: design phase of the CDT, definition phase of partial views and composition phase of global views [1].

1. Design phase of the Context Tree: in this phase, the CDT is designed to identify significant context elements for the considered application. In fact, it focuses on the definition of contexts and on the elements that compose them. These contexts must be identified and shaped, indicating particular elements that characterize each of them. As it has been said, it is available a special tool called CDT to make context design. Various CDT were made for specific environments in order to represent and manage a multitude of different contexts and in order to identify, represent, preserve and make available cultural points for each type of user.

2. Definition phase of partial views: after the definition of all the contexts and their context elements, in this step a different portion of the database is associated to each context element, containing the relevant data for it. In practice, the goal is to find the appropriate value for a given dimension, in order to obtain, by means of the values of all the dimensions, a valid query and specific to the context in which the user is located.

3. Composition phase of global views: this is the phase where you have the automatic generation of views associated with each context, which is made starting from partial views associated with context elements. After the creation of the global views of the contexts, the answers to questions that will be asked to the system will be developed from these views and, in particular, from the view associated with the context in which you are located when the query is performed.

In particular, once defined the values for each dimension, you can use all the information obtained in order to identify the right context and offer contextual resources for the user.

In figure 1, it is shown a general designed CDT, called Meta CDT, which is the starting point for the design of a specific CDT that can be exploited in contextual applications [13]. You may note six top dimensions, which correspond to the questions of the 5W1H method: Location (WHERE), Role (WHO), Time (WHEN), Situation (HOW), Interest (WHAT) and Utilization (WHY). 


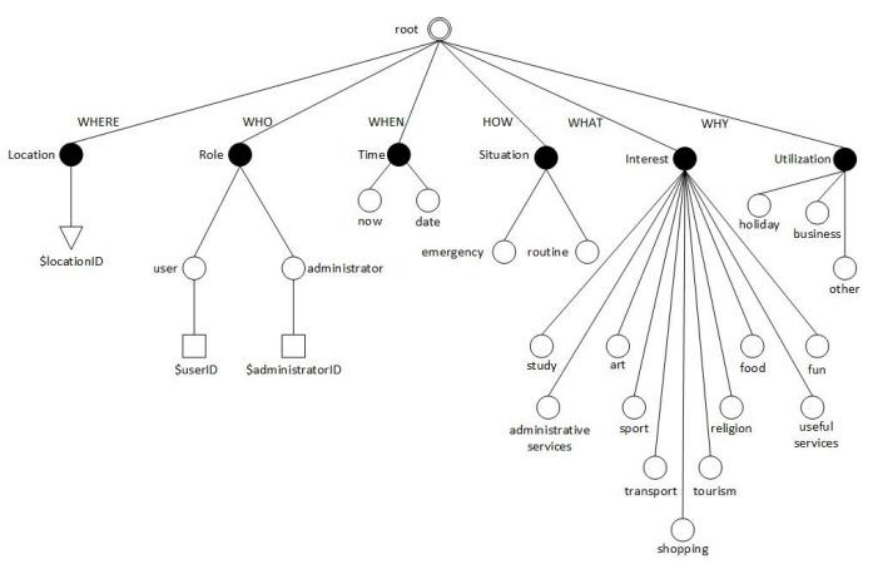

Figure 1. Meta CDT.

In particular, there are two types of users and eleven categories of interests. In this case, as shown in figure 2, a partial view could be related to dimension "Role": once logged in, the application is able to recognize the user and to know more precisely whether he/she is, for example in tourist areas, a resident or a tourist. Thus, the value "tourist" of dimension "Role" is a partial view for the current context: using this knowledge, you can exclude certain resources, not suitable or useful to the tourist role.

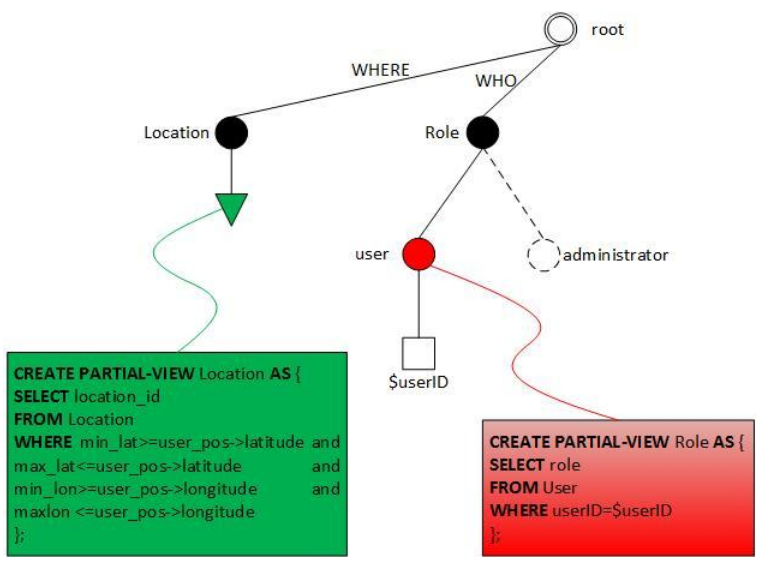

Figure 2. Example of Partial Views.

A context element is defined as an assignment d_name ${ }_{i}$ $=$ value, where $\mathrm{d}$ _namei indicates a possible size or undersize of CDT (it is the label of a dimension node), while value may represent the label of one of the concept nodes that are children of the considered dimension node or the value of a parameter referring to one of these concept nodes or the value of a parameter referring to the considered dimension node.

For example, these assignments are possible context elements:

Interest $=$ "tourism", Location $=$ \$locationID $($ for example, ID = 3), Role = \$userID->role (for example, ID = 15), Utilization = "holiday".

A context is specified as: $\wedge\left(\mathrm{d}\right.$ name $_{i}=$ value $)$ : it is defined as an "and" among different context elements.

Several context elements, combined with each other by means of an "and", damage, therefore, the origin of a context [5]
For example, a possible framework that can be obtained from the previously seen CDT, through the context elements that we have listed, is:

$$
\begin{gathered}
C=(\text { Location }=\$ \text { locationID }(I D=3)) \wedge(\text { Role }= \\
\quad \text { \$userID- }>\text { role }(I D=15)) \\
\wedge(\text { Time }=\text { "now" }) \wedge(\text { Situation }=\text { "routine" })
\end{gathered}
$$

$\wedge($ Interest $=$ "tourism" $) \wedge($ Utilization $=$ "holiday")

The context is defined as a user, interested in tourism, who uses the contextual app on vacation, in a called place.

\section{A CONTEXT AWARE APP FOR PROMOTING TOURISM EVENTS}

In this section, we will present a contextual app designed and implemented according to what was described previously. In particular, we have thought to apply the approach in the context of Artist's Lights Christmas event that every year is held in Salerno (Regione Campania in Italy) and that involves hundreds of thousands of tourists. From November to January, with light installations, some by local artists exclusively for Salerno, scattered through the main streets and in the most beautiful and attractive corners of the city center.

In this phase, we have collected the services and contents potentially useful for the citizens and situate them on the map defining the activation zones (figure 3 ).

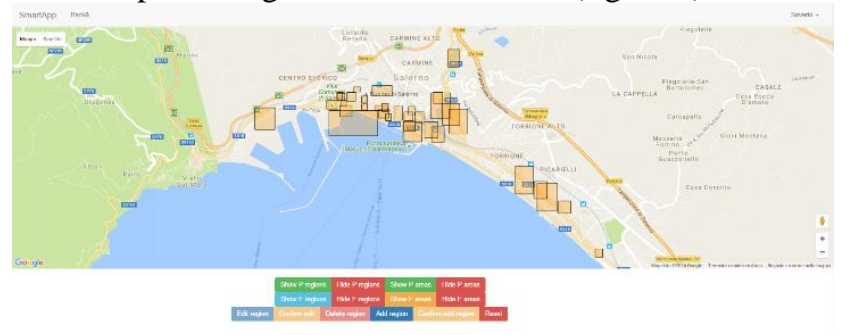

Figure 3. Definition of the activation areas of services and contents

Moreover, we have defined the different typologies of citizens (tourist and expert user) associating them to a previously established set of services and contents. Having the town a series of Christmas contents, we have developed services and contents in support of them too.

All information about places of worship and shops has been uploaded, for any building or area of potential.

The App has been developed with hybrid technologies (Ionic Framework and Apache Cordova) to allow an easier publication both in Android and Apple environment (figure 4).

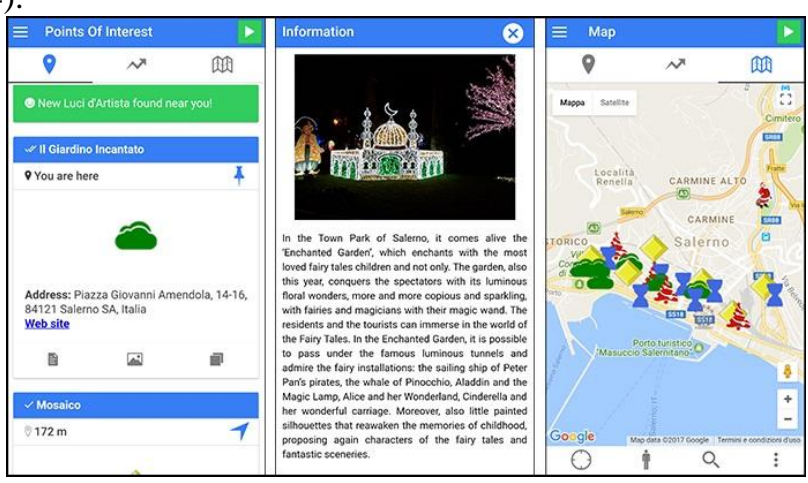

Figure 4. Screenshots with some features of contextual application.

The experimental phase aims to evaluate the proposed contextual model. Initially, the App has been presented to the population in November 2016. They have been involved overall about 2000 tourists between 18 and 60 years old. 
During this event, the app has been installed on the mobile devices of the tourists.

After having interacted for some days with the application, the participants have then answered on the basis of the Likert scale to fourteen statements, divided into four sections. To every question present in the section, five possible answers have been associated: I strongly agree - I agree - Undecided (Neither agree nor disagree) - I disagree- I strongly disagree.

The questionnaire in detail is the following:

Section A: App - Context

- A1. The App gives the user tailor-made contents and services in the right place.

- A2. The App allows the user to know several item of the Artist's Lights.

- A3. The App supplies services according to the interests selected in the user profile.

Section B: App - Further aspects

- B1. Information about each item of Artist's Lights is very useful.

- B2. The contents, such as descriptions and images, are of high quality and represent one of the strong points of Artist's Lights

- B3. The services associated to the items allow a higher immediacy than a classic research on the Internet.

Section C: App - Functionality

- C1. The plan itinerary service allows easily realizing an itinerary in the Artist's Lights according to the user's preferences.

- C2. The explore surroundings service is very useful to know what there is nearby and eventually reach them.

- C3. The functionality of $\mathrm{QR}$ code in inner environments can be well used.

Section D: App - Future developments

- D1. It would be interesting to have a higher integration with the main social networks.

- D2. It would be interesting to insert the available time in the plan itinerary service.

Table 1 presents a synthesis of the answers of the participants to each declaration.

TABLE I: Experimental results

\begin{tabular}{|c|r|r|r|r|r|}
\hline $\begin{array}{c}\text { Likert } \\
\text { Scale }\end{array}$ & $\begin{array}{c}\text { Strongly } \\
\text { agree }\end{array}$ & Agree & $\begin{array}{c}\text { Neither } \\
\text { agree nor } \\
\text { disagree }\end{array}$ & Disagree & $\begin{array}{c}\text { Strongly } \\
\text { disagree }\end{array}$ \\
\hline A1 & 698 & 996 & 186 & 69 & 51 \\
\hline A2 & 941 & 723 & 187 & 89 & 60 \\
\hline A3 & 732 & 966 & 207 & 54 & 41 \\
\hline B1 & 698 & 974 & 148 & 102 & 78 \\
\hline B2 & 798 & 845 & 234 & 78 & 45 \\
\hline B3 & 784 & 969 & 196 & 33 & 18 \\
\hline C1 & 1012 & 771 & 124 & 44 & 49 \\
\hline C2 & 824 & 942 & 112 & 69 & 53 \\
\hline C3 & 781 & 899 & 199 & 74 & 47 \\
\hline D1 & 835 & 818 & 196 & 87 & 64 \\
\hline D2 & 829 & 745 & 287 & 87 & 52 \\
\hline
\end{tabular}

As shown in this table, of the 2000 participants who have interacted with the application, many agree and/or strongly agree that the system gives appropriate contextual information about the place, further aspects and functionality are very useful and future developments are interesting. Instead, only in few cases, the participants do not are particularly satisfied.

As can noticed from the figure 5, users show great appreciation for the app. In general, they appreciated the proposed contents and services.

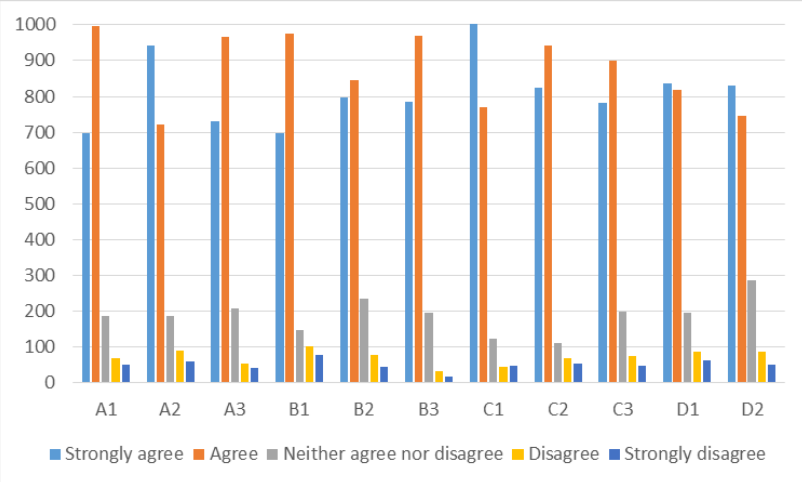

Figure 5. Graphic analysis of experimental results.

\section{CONCLUSIONS}

Based on the concept of Context Dimention Tree, in this paper is presented the use of Context-Aware approach in order to allow contextual decisions for users according to his/her needs. CDT is a graphical formalism able to model a context by the approach of the $5 \mathrm{~W} 1 \mathrm{H}$ method.

To obtain this results, a mobile application was developed keeping track of users needs, based on context, providing personalized services. The App bases its 'contextual' functioning on the adoption of the CDT that is able to shape the context and the actions to implement.

The App has been designed for the Christmas event, Artist's Lights, which was held in Salerno, a city in the south of Italy. The following activities have as purpose the application of the proposed methodology to more complex environments, for dimension and number of potential places to manage.

\section{REFERENCES}

[1] G. Annunziata, F. Colace, M. De Santo, S. Lemma, and M. Lombardi, "ApPoggiomarino: A Context Aware App for eCitizenship," The 18th International Conference on Enterprise Information Systems (ICEIS), 2016.

[2] J. Barreira , M. Bessa, L. Barbosa and L. Magalhaes, "A ContextAware Method for Authentically Simulating Outdoors Shadows for Mobile Augmented Reality, 'IEEE Transactions on Visualization and Computer Graphics, Volume: PP, Issue: $99,2017$.

[3] J. Beysens, A. Chiumento, S. Pollin and M. Li, "TouchSpeaker, a Multi-Sensor Context-Aware Application for Mobile Devices," IEEE International Workshop on Signal Processing Systems, 2016.

[4] C. Bolchini, C. Curino, E. Quintarelli, F.A. Schreiber, and L. Tanca, "A survey of context models and a preliminary methodology for context driven data view definition," Technical Report 4.1, ESTEEM Project, 2006.

[5] M. Casillo, L. Cerullo, F. Colace, S. Lemma, M. Lombardi, and A. Pietrosanto, "An Adaptive Context Aware App for the Tourism," In Proceedings of the The 3rd Multidisciplinary International Social Networks Conference on SocialInformatics 2016, Data Science 2016 (p. 26). ACM, 2016.

[6] M. Casillo, F. Colace, S. Lemma, M. Lombardi, and A. Pietrosanto, "An Ontological Approach to Digital Storytelling," In Proceedings of the The 3rd Multidisciplinary International Social Networks Conference on SocialInformatics 2016, Data Science 2016 (p. 27). ACM, 2016.

[7] F. Colace, M. De Santo, L. Greco, and P. Napoletano, "Weighted Word Pairs for query expansion," Information Processing and Management, 51 (1), pp. 179-193, 2015. 
[8] F. Colace, P. Foggia, and G. Percannella, "A probabilistic framework for TV-news stories detection and classification," IEEE International Conference on Multimedia and Expo, ICME 2005, 2005, art. no. 1521680, pp. 1350-1353, 2005.

[9] F. Colace, M. De Santo, and L. Greco, "An adaptive product configurator based on slow intelligence approach," International Journal of Metadata, Semantic and Ontologies (IJMSO), Vol.9, No.2, pp. 128-137, 2014.

[10] F. Colace, L. Greco, S. Lemma, M. Lombardi, D. Yung, and S.K Chang, "An Adaptive Contextual Recommender System: a Slow Intelligence Perspective," The Twenty-Seventh International Conference on Software Engineering and Knowledge Engineering (SEKE), pp. 64-71, 2015.

[11] F. Colace, M. De Santo, L. Greco, V. Moscato, and A. Picariello, “A collaborative user-centered framework for recommending items in Online Social Networks," Computers in Human Behavior 51: pp. 694-704, 2015.

[12] F. Colace, M. De Santo, S. Lemma, M. Lombardi, A. Rossi, A. Santoriello, A. Terribile, and M. Vigorito, "How to Describe Cultural Heritage Resources in the Web 2.0 Era?," Proceedings - 11th International Conference on Signal-Image Technology and InternetBased Systems (SITIS), art. no. 7400656, pp. 809-815, 2015.

[13] F. Colace, L. Greco, S. Lemma, M. Lombardi, F. Amato, V. Moscato, and A. Picariello, "Contextual Aware Computing and Tourism: A Case Study," The Eleventh International Conference on Signal-Image Technology \& Internet-Based Systems (SITIS), pp. 804-808, 2015

[14] R. Martoglia, "AMBIT: Semantic Engine Foundations for Knowledge Management in Context-dependent Applications," Proc. of the 27th International Conference on Software Engineering and Knowledge Engineering (SEKE), 2015.

[15] M. Masango, F. Mouton, A. Nottingham and J. Mtsweni, "Context Aware Mobile Application for Mobile Devices," Information Security for South Africa (ISSA), 2016.

[16] N. Mendes, B. Alves and S. Paiva, "Context-aware mobile recommender system based on activity patterns," 17th IEEE International Conference on Mobile Data Management, 2016.

[17] R. Morales, B. Igler, S. Bohm and P. Chitchaipoka, "Context-Aware Mobile Language Learning," 12th International Conference on Mobile Systems and Pervasive Computing, MobiSPC, 2015.

[18] C. Parent, K. Schewe, V. Storey, and B. Thalheim, Conceptual Modeling - ER 2007, 26th International Conference on Conceptual Modeling, Auckland, New Zealand, November 5-9, 2007.

[19] Y. S. Rawat and M. S. Kankanhalli, "ClickSmart: A Context-Aware Viewpoint Recommendation System for Mobile Photography," IEEE Transactions on Circuits and Systems for Video Technology, vol. 27, no. 1, 2017.

[20] R. Roostaei and Z. Movahedi, "Mobility and Context-Aware Offloading in Mobile Cloud Computing," IEEE Conferences on Ubiquitous Intelligence \& Computing, Advanced and Trusted Computing, Scalable Computing and Communications, Cloud and Big Data Computing, Internet of People, and Smart World Congress, 2016.

[21] L. Tanca, C. Bolchini, C. Curino, and F.A. Schreiber, "Context integration for mobile data tailoring," Italian Symposium on Database Systems (SEBD), pp. 48-55, 2006

[22] N. M. Villegas and H. A. Mller, "Managing dynamic context to optimize smart interactions and services," The Smart Internet, volume 6400 of Lecture Notes in Computer Science, pages 289- 318. Springer Berlin Heidelberg, 2010.

[23] Z. Xiang, S. Deng, S. Liu, B. Cao and J. Yin, "CAMER: A ContextAware Mobile Service Recommendation System," IEEE International Conference on Web Services, 2016.

[24] Z. Zhao, H. Chen, R. Li and Z. Wang, "An Approach to Contextaware Service Pushing for Mobile Computing," IEEE International Conference on Mobile Services, 2016. 